\title{
Baroreflex and chemoreflex dysfunction in streptozotocin-diabetic rats
}

\author{
P. Dall'Ago ${ }^{1}$, \\ T.G. Fernandes ${ }^{1}$, \\ U.F. Machado ${ }^{2}$, \\ A.A. Belló ${ }^{1}$ and \\ M.C. Irigoyen ${ }^{1}$
}

\author{
${ }^{1}$ Laboratório de Fisiologia Cardiovascular, Departamento de Fisiologia, \\ Instituto de Biociências, Universidade Federal do Rio Grande do Sul, \\ 90050-170 Porto Alegre, RS, Brasil \\ ${ }^{2}$ Departamento de Fisiologia, Instituto de Ciências Biomédicas, \\ Universidade de São Paulo, 05508-900 São Paulo, SP, Brasil
}

\author{
Correspondence \\ M.C. Irigoyen \\ Laboratório de Fisiologia \\ Cardiovascular \\ Departamento de Fisiologia \\ IB, UFRGS \\ Rua Sarmento Leite, 500 \\ 90050-170 Porto Alegre, RS \\ Brasil \\ Fax: 55 (051) 226-7191 \\ E-mail: mcci@vortex.ufrgs.br \\ Presented at the XI Annual Meeting \\ of the Federação de Sociedades de \\ Biologia Experimental, Caxambu, \\ MG, Brasil, August 21-24, 1996.
}

Research supported by CAPES, CNPq, FINEP and PROPESP/UFRGS. Publication supported by FAPESP.

Received April 19, 1996 Accepted November 6, 1996

\begin{abstract}
Several investigators have demonstrated that streptozotocin (STZ) diabetes induces changes in the autonomic control of the cardiovascular system. Changes in cardiovascular function may be related to peripheral neuropathy. The aim of the present study was to analyze changes in heart rate (HR) and arterial pressure (AP) as well as baroreflex and chemoreflex sensitivity in STZ-induced diabetic male Wistar rats (STZ, $50 \mathrm{mg} / \mathrm{kg}, i v, 15$ days). Intra-arterial blood pressure signals were obtained for control and diabetic rats ( $\mathrm{N}=9$, each group). Data were processed in a data acquisition system (CODAS, $1 \mathrm{kHz}$ ). Baroreflex sensitivity was evaluated by measuring heart rate changes induced by arterial pressure variation produced by phenylephrine and sodium nitroprusside injection. Increasing doses of potassium cyanide $(\mathrm{KCN})$ were used to evaluate bradycardic and pressor responses evoked by chemoreflex activation. STZ induced hyperglycemia (447 \pm 49 vs $126 \pm 3 \mathrm{mg} / \mathrm{dl})$, and a reduction in AP $(99 \pm 3$ vs $118 \pm 2$ $\mathrm{mmHg})$, resting HR (296 \pm 11 vs $355 \pm 16 \mathrm{bpm})$ and plasma insulin levels $(16 \pm 1$ vs $57 \pm 11 \mu \mathrm{U} / \mathrm{ml})$. We also observed that the reflex bradycardia $(-1.68 \pm 0.1 v s-1.25 \pm 0.1 \mathrm{bpm} / \mathrm{mmHg}$, in the diabetic group) and tachycardia $(-3.68 \pm 0.5 v s-1.75 \pm 0.3 \mathrm{bpm} / \mathrm{mmHg}$, in the diabetic group) produced by vasopressor and depressor agents were impaired in the diabetic group. Bradycardia evoked by chemoreflex activation was attenuated in diabetic rats (control: $-17 \pm 1,-86 \pm 19$, $-185 \pm 18,-208 \pm 17$ vs diabetic: $-7 \pm 1,-23 \pm 5,-95 \pm 13,-140 \pm 13$ bpm), as also was the pressor response (control: $6 \pm 1,30 \pm 7,54 \pm 4$, $59 \pm 5$ s diabetic: $6 \pm 1,8 \pm 2,33 \pm 4,42 \pm 5 \mathrm{mmHg}$ ). In conclusion, the cardiovascular responses evoked by baroreflex and chemoreflex activation are impaired in diabetic rats. The alterations of cardiovascular responses may be secondary to the autonomic dysfunction of cardiovascular control.
\end{abstract}

Key words

- Streptozotocin

- Experimental diabetes

- Baroreflex sensitivity

- Chemoreflex sensitivity

- Autonomic dysfunction 
Patients with diabetes mellitus are particularly prone to disorders related to the control of the cardiovascular system, including microangiopathy, atherosclerosis, hypertension and autonomic neuropathy (1). Diabetics with abnormal cardiovascular reflexes have a higher incidence of mortality than diabetics with normal autonomic reflex function (2). The arterial baroreceptor exerts a major influence on both the sympathetic and parasympathetic limbs of the autonomic nervous system and cardiovascular function. Several investigators have studied the baroreflex function in hyperglycemic rats treated with streptozotocin (1,3-5). The reflex tachycardic response elicited by arterial pressure (AP) reduction was found to be attenuated in short-term diabetes (5), while the reflex bradycardia in response to an AP increase has been reported to be normal in short-term diabetes. In contrast, baroreflexmediated bradycardia during increasing AP is impaired in alloxan-induced diabetic rabbits at the same time that reflex tachycardia to AP reduction is preserved (6). Jackson and Carrier (1) and Homma et al. (4) have reported an enhancement in baroreflex function when vasopressor or depressor agents are exogenously administered and when the right cervical sympathetic or vagus nerve is electrically stimulated, respectively. Several lines of evidence indicate that changes in baroreflex function are probably due to peripheral dysfunction of the autonomic nervous system in diabetic patients and animals $(1,4,6)$.

The peripheral chemoreceptors have been less studied than the baroreceptors, but they represent an important group of afferents that control the autonomic function in mammals. The peripheral chemoreceptors reflexely control respiration as well as circulation (7). The cardiovascular responses evoked by chemoreceptor stimulation induced by intravenous injections of potassium cyanide $(\mathrm{KCN})$ are a complex result of the primary activation of sympathetic and parasympathetic neurons, resulting in characteristic cardiovascular (bradycardia and hypertension), respiratory (tachypnea) and behavioral (alerting) responses (8). The peripheral chemoreceptors have been implicated in the pathogenesis of autonomic changes as observed in obstructive sleep apnea and hypertension $(9,10)$. In addition, Homma et al. (11) demonstrated impairment in the sensitivity of peripheral or central chemoreceptors in diabetic patients. Also, the close anatomical and functional relationship between chemoreceptors and baroreceptors raises the possibility that the impairment of baroreflex function may be associated with an impairment of the chemoreflex, since chemoreflex and baroreflex are integrated by a common neuronal network $(8,12)$.

The purpose of the present study was to analyze changes in heart rate (HR) and AP as well as baroreflex and chemoreflex sensitivities in streptozotocin (STZ)-induced diabetic rats 15 days after diabetes induction.

The experiments were performed on male Wistar rats weighing $216.6 \pm 8 \mathrm{~g}, \mathrm{~N}=9$ (controls) and 226.6 $\pm 8 \mathrm{~g}, \mathrm{~N}=9$ (diabetics), housed in individual cages with free access to water and food. Rats were made diabetic by a single injection of STZ $(50 \mathrm{mg} / \mathrm{kg}, i v$, Sigma Chemical Co., St. Louis, MO) dissolved in $10 \mathrm{mM}$ citrate buffer, $\mathrm{pH} 4.5$. The rats were fasted for $8 \mathrm{~h}$ before STZ injection. One day before the experiment, two catheters (PE-10) were implanted into the femoral artery and vein, under ether anesthesia, for direct measurements of mean arterial pressure (MAP) and for drug administration, respectively. The arterial catheter was attached to 20-cm PE-90 tubing that was connected to a pressure transducer $(\mathrm{P} 23 \mathrm{Db}$, Gould-Statham, Oxnard, CA). Fifteen days after STZ administration blood pressure signals were recorded for 40 min with a microcomputer equipped with an analog-to-digital converter board (CODAS, $1 \mathrm{kHz}$, Dataq Instruments, Akron, $\mathrm{OH})$. The recorded data were analyzed on a beat-to-beat basis to 
quantify changes in MAP and HR. The venous catheter was connected to an extension (PE-10) for drug infusion when necessary. After a control MAP recording the animals remained quiet for $10-15 \mathrm{~min}$ and the following tests were performed: 1) baroreflex sensitivity testing by intravenous injections of increasing doses of phenylephrine $(0.25-32 \mu \mathrm{g} / \mathrm{ml})$ and sodium nitroprusside $(0.05-1.6 \mu \mathrm{g} / \mathrm{ml})$ given sequentially $(0.1$ $\mathrm{ml}$ ) as bolus injections, and 2) chemoreflex sensitivity testing by intravenous injections of $\mathrm{KCN}$. Increasing doses of $\mathrm{KCN}(60,100$, 140 and $180 \mu \mathrm{g} / \mathrm{kg}$ ) were injected into control and diabetic rats after the cardiovascular parameters had returned to basal levels. Peak increases or decreases in MAP after phenylephrine or sodium nitroprusside injections and the corresponding peak reflex changes in HR were recorded for each dose of the drug. Baroreflex sensivity is reported as values derived from fitting a regression line through points corresponding to all changes in HR related to the induced changes in MAP. A time interval between doses was necessary for blood pressure to return to baseline. After animals had remained quiet for 10-15 min the chemoreflex function was evaluated by increasing intravenous doses of $\mathrm{KCN}$. To analyze the responses, AP and HR were measured continuously for $10 \mathrm{sec}$ before and for $15 \mathrm{sec}$ after the injections. Subsequent doses of $\mathrm{KCN}$ were injected after recovery of the cardiovascular parameters and when the animal was quiet again. One hour before recording AP pulses, blood samples $(1 \mathrm{ml})$ were collected through the arterial catheter to measure basal glucose and insulin levels, using a colorimetric enzymatic test (Enz color, Bio Diagnostica) and radioimmunoassay (Pharmacia), respectively. To prevent hypovolemia after the collection of blood samples, both control and STZ-treated rats were injected intravenously with $1 \mathrm{ml}$ of saline.

Data are reported as means \pm SEM and the unpaired Student $t$-test was used to com-

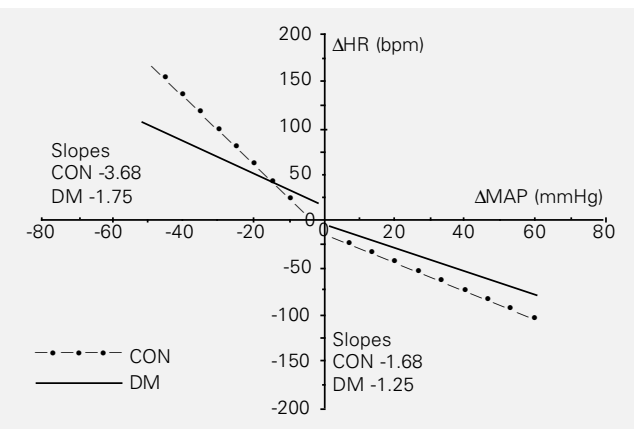

pare values between groups. Baroreflex sensitivity was evaluated by regression analysis of different groups and the slopes were compared using the $t$-test for unpaired data. Differences between controls and diabetic rats in $\mathrm{AP}$ and $\mathrm{HR}$ responses to $\mathrm{KCN}$ were compared by analysis of variance for repeated measures and the post-hoc test employed was the Newman-Keuls test. Statistical significance was set at the 0.05 level in all procedures.

All nine rats given STZ developed severe hyperglycemia $(126 \pm 3 v s 447 \pm 49 \mathrm{mg} / \mathrm{dl}$ for the diabetic group, $\mathrm{P}=0.001$ ) accompanied by a decrease in body weight $(264 \pm 5 \mathrm{vs}$ $198 \pm 8 \mathrm{~g}$ for the diabetic group, $\mathrm{P}=0.001$ ) and plasma insulin levels (57 \pm 11 vs $16 \pm 1$ $\mu \mathrm{U} / \mathrm{ml}$ for the diabetic group, $\mathrm{P}=0.001$ ). The 15-day STZ-induced diabetes group had significantly reduced systolic AP $(140 \pm 2 v s$ $118 \pm 3 \mathrm{mmHg}$ for the diabetic group, $\mathrm{P}=$ $0.001)$, diastolic AP $(99 \pm 3$ vs $81 \pm 4 \mathrm{mmHg}$ for the diabetic group, $\mathrm{P}=0.001$ ) and MAP (118 \pm 2 vs $99 \pm 3 \mathrm{mmHg}$ for the diabetic group, $\mathrm{P}=0.001)$. Resting HR decreased significantly in diabetic rats as compared to control rats $(355 \pm 16$ vs $296 \pm 11$ bpm for the diabetic group, $\mathrm{P}=0.010$ ). Figure 1 shows the HR responses evoked by pressor and depressor agents (phenylephrine and sodium nitroprusside, respectively). Reflex bradycardia elicited by phenylephrine was significantly reduced in the diabetic group $(-1.68 \pm 0.1 v s-1.25 \pm 0.1 \mathrm{bpm} / \mathrm{mmHg}$ in the diabetic group, $\mathrm{P}=0.019)$. Reflex tachycardia elicited by sodium nitroprusside was also
Figure 1 - STZ-diabetic rats presented an impairment of bradycardic (lower right) and tachycardic (upper left) response to AP changes induced by increasing doses of phenylephrine and sodium nitroprusside. The slope of the lines obtained by linear regression analysis was -1.68 vs $-1.25 \mathrm{bpm} / \mathrm{mmHg}(\mathrm{P}=$ 0.019 ) for control (CON) and diabetic rats (DM), respectively, after an increase in AP. The slope of the lines obtained by linear regression analysis was $-3.68 \mathrm{vs}$ $-1.75 \mathrm{bpm} / \mathrm{mmHg}(P=0.004)$ for control and diabetic rats, respectively, after a decrease in AP (unpaired Student $t$-test). 
significantly reduced in the diabetic group, as indicated by the slope of the regression line relating changes in HR to changes in $\operatorname{MAP}(-3.68 \pm 0.5 v s-1.75 \pm 0.3 \mathrm{bpm} / \mathrm{mmHg}$ in the diabetic group, $\mathrm{P}=0.004)$. The bradycardia evoked by chemoreflex activation produced by increasing doses of $\mathrm{KCN}$ was markedly impaired in diabetic rats (control: $-17 \pm$ $1,-86 \pm 19,-185 \pm 18,-208 \pm 17$ vs diabetic: $-7 \pm 1,-23 \pm 5,-95 \pm 13,-140 \pm 13 \mathrm{bpm}, \mathrm{P}=$ 0.001 , Figure 2A). The pressor responses evoked by the chemoreflex were also markedly decreased in the diabetic group (control: $6 \pm 1,30 \pm 7,54 \pm 4,59 \pm 5$ vs diabetic: $6 \pm 1,8 \pm 2,33 \pm 4,42 \pm 5 \mathrm{mmHg}, \mathrm{P}=0.010$, Figure 2B).

The major findings of the present study are that 15-day STZ-diabetic rats showed 1) decreased AP and HR, 2) depressed bradycardic and tachycardic responses activated by the baroreflex, and 3) impaired bradycardic and pressor responses evoked by chemoreflex activation with KCN. Moreover, STZ induced hyperglycemia, reduction in body weight and insulinopenia.

Diabetic rats had significantly lower resting MAP and HR in the conscious state. Jackson and Carrier (1) suggested that the decrease in AP may be the result of a decreased cardiac output in diabetic rats due to hypovolemia caused by hyperglycemic osmotic diuresis. However, Cohen et al. (13) observed that the diabetic animals were polyuric with a high urine flow, reflecting

Figure 2 - Line graphs showing the effects of streptozotocin-induced diabetes on mean arterial pressure (MAP) responses (A) and heart rate $(\mathrm{HR})$ responses (B) of control $(\bullet, N=9)$ and diabetic $(\square, N=9)$ rats to increasing doses of KCN. Data are reported as means \pm SEM. ${ }^{*} \mathrm{P}<0.05 \mathrm{com}-$ pared to control (ANOVA).

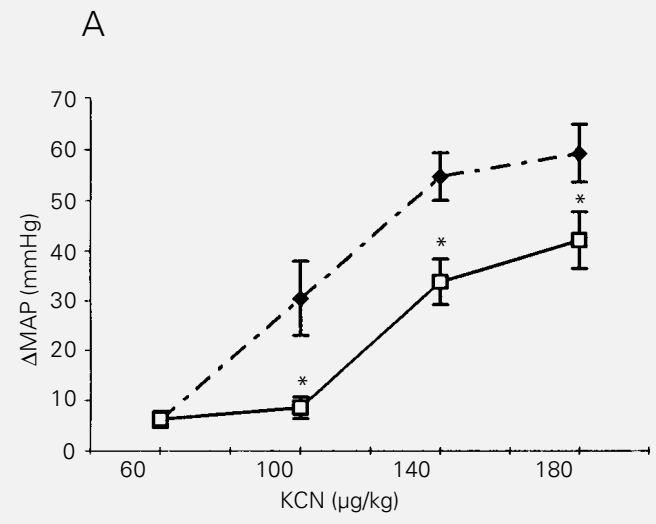

the osmotic diuretic effect of glucose. In these experiments, the renal physiologic parameters demonstrated increased plasma volume, presumably related to osmotic effects of glucose resulting in both expansion of extracellular fluid volume and induction of diuresis (13). In the present study, the collection of blood samples may have contributed to the changes in AP, although the rats were injected with equal volumes of saline after collection. These data are in accordance with our previous studies $(5,14)$ that showed a reduction in AP in STZ-induced diabetes measured before and/or after blood sample collection. The observed hypotension may also have been due to an increase in parasympathetic outflow in diabetic rats, although Maeda et al. (14) demonstrated a reduction in vagal function 5 days after STZ treatment, suggesting that changes in arterial blood pressure are not related to an increase in parasympathetic outflow. However, differences in baroreflex sensitivity at different times of diabetes $(3,4)$ suggest changes in the ability to regulate HR, including changes in parasympathetic $(1,3)$ and sympathetic control (4). In contrast, previous data from our laboratory (5) showed impairment of the vasopressor response to phenylephrine, indicating that changes in peripheral resistance may contribute to AP reduction. Although the reduction in heart rate may be attributed to a change in the sinoatrial node (14), functional changes in

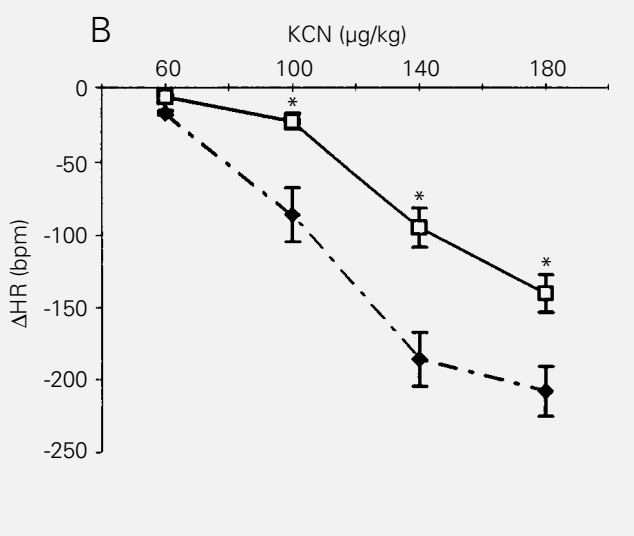


cholinergic mechanisms cannot be excluded as a causal factor. Moreover, the decrease in heart rate may also be due to changes in Breceptors in the heart. Savarese and Berkowitz (15) observed bradycardia in STZ-diabetic rats probably related to a decrease in the number of ventricular $\beta$-receptor binding sites in the diabetic rat myocardium.

It has been demonstrated that short-term diabetes induces impairment of baroreflexmediated tachycardia in response to a decrease in AP at the same time that reflex bradycardia in response to an increased AP is preserved (5). In contrast, other studies $(1,4)$ demonstrated enhanced sensitivity of baroreceptor reflex in diabetic rats. McDowell et al. (6) demonstrated that baroreflex-mediated bradycardia is impaired while reflex tachycardia is preserved in diabetic rabbits. The impairment in bradycardic and tachycardic responses to changes in AP observed in the present study indicates that changes in baroreflex sensitivity may be related to time-dependent changes caused by metabolic disorders due to a hyperglycemic state or insulinopenia. The present study involving a streptozotocin-induced rat model of diabetes is an attempt to investigate mechanisms of baroreflex dysfunction in 15-day STZ diabetes. The controversial data regarding changes in baroreflex sensitivity after diabetes may be attributed to differences in the experimental design as well as in the analytical methodology $(5,12)$, suggesting that these differences may be correlated with the time course of diabetes. Indeed, not only the volume status of rats but also factors other than plasma volume may be altered during diabetes (13) and, consequently, the osmotic diuresis may be different at each time after STZ injection. Moreover, the animal model and the diabetogen used could change the interpretation of the data (6).

Franchini and Krieger (8) demonstrated that the cardiovascular responses (HR and AP) evoked by chemoreflex activation induced by $\mathrm{KCN}$ in conscious rats mostly de- pend on cardiac-vagal activation (bradycardia), with a contribution by the cardiac-sympathetic activation to determine the final pressor responses. Here we demonstrated impairment of cardiovascular responses evoked by chemoreflex activation by KCN in diabetic rats. Based on these data, we suggest that the impairment of chemoreflex sensitivity in diabetic rats may be related to the reduction of cardiac-vagal function. It is not clear whether these alterations are related to specific changes in the peripheral chemoreceptor afferent limb or to central cardiorespiratory integration of the reflex arc. Another finding of the present study was the reduction in pressor responses to chemoreflex activation in the diabetic group. This reduced vascular response as well as the impairment of baroreflex sensitivity may be related to a decrease in peripheral sympathetic activity $(5,8)$. Morphological changes in axons from the sympathetic paravertebral chain reported by Monckton and Pehowich (16) in streptozotocin-treated rats may contribute to the decreased pressor responses evoked by chemoreflex activation. Moreover, the interactions between the sympathetic and parasympathetic systems are complex as demonstrated previously (17), showing that the effects of vagal stimulation on HR are enhanced when the cardiac sympathetic nerve fibers are simultaneously stimulated, suggesting a different vagal activation at different levels of sympathetic function. On the other hand, the changes in the baroreflex and chemoreflex function seem to be related to changes in the target organs, since basal MAP and HR were significantly reduced in diabetic rats. These alterations may be related to changes in sympathetic and parasympathetic activity or to changes in the receptors in the vessels and the heart as described elsewhere (15).

In conclusion, decreased baroreceptor sensitivity and impairment of the chemoreflex function may be due to dysfunction of the autonomic nervous system. Changes in 
sympathetic and parasympathetic branches to the heart could explain the decrease in baroreflex and chemoreflex function. The central nervous system may also contribute to this impairment since the afferent limb of the baro- and chemoreceptor seems to be operating normally (18). However, the exact site of the defect in the reflex arc was not determined. Additional data will be necessary to evaluate the participation of the central nervous system and the efferent limb of the reflex arc.

\section{References}

1. Jackson CV \& Carrier GO (1983). Influence of short-term experimental diabetes on blood pressure and heart rate in response to norepinephrine and angiotensin II in the conscious rat. Journal of Cardiovascular Pharmacology, 5: 260-265.

2. Ewing DJ, Campbell IW \& Clark BF (1980). The natural history of diabetic autonomic neuropathy. Quarterly Journal of Medicine, 49: 95-108.

3. Chang KSK \& Lund DD (1986). Alterations in the baroreceptor reflex control of heart rate in streptozotocin diabetic rats. Journal of Molecular and Cellular Cardiology, 18: 617-624.

4. Homma S, Yamazaki $Y$ \& Karakida $T$ (1993). Blood pressure and heart rate relationships during cervical sympathetic and vagus nerve stimulation in streptozotocin diabetic rats. Brain Research, 629: 342-344.

5. Maeda CY, Fernandes TG, Lulhier F \& Irigoyen MC (1995). Streptozotocin modifies arterial pressure and baroreflex sensitivity in rats. Brazilian Journal of Medical and Biological Research, 28: 497-501.

6. McDowell TS, Chapleau MW, Hajduczok G \& Abboud FM (1994). Baroreflex dysfunction in diabetes mellitus. I. Selective impairment of parasympathetic control of the heart rate. American Journal of Physiology, 266: H235- 243.
7. Marshall JM (1994). Peripheral chemoreceptors and cardiovascular regulation. Physiological Reviews, 74: 543-594.

8. Franchini KG \& Krieger EM (1993). Cardiovascular responses of conscious rats to carotid body chemoreceptor stimulation by intravenous KCN. Journal of the Autonomic Nervous System, 42: 63-70.

9. Fletcher EC, Lesske K, Qian W, Miller III CC \& Unger T (1992). Repetitive episodic hypoxia causes diurnal elevation in blood pressure in rats. Hypertension, 19: 555561.

10. Trezbsky A (1992). Arterial chemoreceptor reflex and hypertension. Hypertension, 19: $562-566$

11. Homma I, Kageyama S, Nagai T, Taniguchi I, Sakai T \& Abé M (1981). Chemosensitivity in patients with diabetic neuropathy. Clinical Science, 61: 599-603.

12. Heistad DD, Abboud FM, Mark AL \& Schmid PG (1974). Interaction of baroreceptor and chemoreceptor reflexes: modulation of the chemoreceptor reflex by changes in baroreceptor activity. Journal of Clinical Investigation, 53: 1226-1236.

13. Cohen AJ, McCarthy DM \& Rossetti RR (1986). Renin secretion by the spontaneously diabetic rat. Diabetes, 35: 341-346.
14. Maeda CY, Fernandes TG, Timm HB \& Irigoyen MC (1995). Autonomic dysfunction in short-term experimental diabetes. Hypertension, 26: 1100-1104

15. Savarese JJ \& Berkowitz BA (1979). ßAdrenergic receptor decrease in diabetic rat hearts. Life Sciences, 25: 2075-2078.

16. Monckton G \& Pehowich E (1980). Autonomic neuropathy in the streptozotocin diabetic rat. Canadian Journal of Neurological Sciences, 7: 135-142.

17. Chassaing C, Duchene-Maruallaz P \& Veyrac J (1983). Effects of catecholamines on cardiac chronotropic response to vagal stimulation in the dog. American Journal of Physiology, 245: H721-H724.

18. Fazan Jr R, Irigoyen MC, Moraes MFD, Maeda CY \& Salgado HC (1995). Baroreceptor function in short-term streptozotocin diabetic rats. Hypertension, 25: 45A. 